\title{
Ontario primary care models: a descriptive study
}

\author{
Logan McLeod PhD, Gioia Buckley MA, Arthur Sweetman PhD
}

Abstract

Background: Between 2001 and 2006, the Ontario government introduced a menu of new primary care models, with elements such as patient enrolment and minimum group sizes, and various combinations of fee-for-service, capitation, pay-for-performance and salary. From the statistical perspective of physicians, as opposed to patients, we looked at the distribution of physician characteristics, group size and patient visit patterns across models to describe primary care practice in Ontario.

Methods: Using administrative data for fiscal year 2010/11 containing information on physician characteristics, patient rostering status, patient visits and other practice information, we described similarities and differences across primary care models.

Results: Our sample included 11626 family physicians. Compared with physicians in the new primary care models, physicians in fee-for-service models are much more likely to work part-time and many, particularly younger and female physicians, do not work in full-year full-scope practices. Among the new primary care models, physicians in capitated models are slightly younger, are less likely to be an international medical graduate, work in smaller physician teams and do not practice in urban areas. On average, physicians saw and rostered 1888 patients. Although there is still substantial variation within each model, fee-for-service physicians saw the fewest patients; physicians in capitated models saw somewhat more, and those in the noncapitated models saw the most patients.

Interpretation: Practice and physician characteristics vary systematically across models. A high percentage of rostered patients see physicians outside the group with which they are rostered. Group-based primary care models may not have a large impact on group integration and continuity in the provision of primary care services.

$\mathrm{B}$ etween 2001 and 2006, the Ontario government introduced a menu of new primary care models characterized by requirements such as patient enrolment (i.e., rostering), group-based care and physician remuneration comprised of various combinations of fee-for-service, capitation, pay-for-performance and salary. Group practice of 3 or more physicians is required in almost all new primary care models, as is the provision of after-hours services. ${ }^{1-3}$ This was a dramatic change in Ontario's primary care landscape. For example, in fiscal year $2000 / 01$, more than $98 \%$ of family physicians were paid using fee-for-service alone. ${ }^{4}$ However, by $2010 / 11$, only $35.2 \%$ of physicians were paid through fee-forservice, with the remainder in a new primary care model (for at least part of the year). The new models are voluntary and, barring a current entry cap of 20 physicians per month for capitation-based models, Ontario physicians can join at any time. As of March 2011, close to 10 million Ontario Health Insurance Plan (OHIP) beneficiaries were rostered.

Although research is ongoing regarding primary health care and physician payment methods, little is known about the basic characteristics of the different primary care models, such as the characteristics of physicians, number of patients by enrolment status, number of visits per patient or percentage of patients who see only their rostering physician/group.
Two recent studies provide some insight; however, they either present aggregate statistics (across an 8-year period and payment models $)^{5}$ or exclude a nontrivial number of nontraditional fee-for-service physicians. ${ }^{6} \mathrm{We}$ sought to fill this gap by describing, from the statistical perspective of Ontarian physicians, differences across the most common primary care models for all physicians.

\section{Methods}

\section{Study design and setting}

We conducted a cross-sectional study of the population of family physicians in Ontario who billed OHIP, by primary

Competing interests: Logan McLeod, Gioia Buckley and Arthur Sweetman report receiving grants from the Government of Ontario and Ontario SPOR Support Unit. No other competing interests were declared.

This article has been peer reviewed.

Correspondence to: Gioia Buckley, buckle@mcmaster.ca

CMAJ Open 2016. DOI:10.9778/cmajo.20160069 
care model, for fiscal year 2010/11. A single fiscal year was employed because it corresponds to the relevant period for payment for models with respect to various maximums, incentives and bonus payments. We used fiscal year 2010/11 as it was the most recent data to which we had access.

\section{Data sources}

Three administrative databases were linked: the Corporate Provider Database provides data on physicians (age, sex, country of medical school graduation, primary care model affiliation, rurality); the OHIP database lists physician claims; and the Client Agency Program Enrollment database covers rostering. All individual patient and physician identifiers were encrypted by the Ontario Ministry of Health and Long-Term Care to anonymize the data. These data are the only administrative data source for primary care use in Ontario and are frequently used in analyses due to their comprehensiveness. ${ }^{2,6}$

\section{Participants}

We use the term "physician" to refer to both general practitioners and physicians with a specialty in family medicine as listed in the Corporate Provider Database. We restricted the sample to active physicians who either billed at least Cdn $\$ 1$ for 1 of the 3 most common fee codes billed (A007 [intermediate assessment], A001 [minor assessment] or A003 [general assessment]), or (ii) rostered at least 1 patient. We deleted a very small number of physicians with practice sizes of more than 10000 and with a percentage of total billing from the 3 most common fee codes that was below $20 \%$.

\section{Variables}

We categorized primary care models into 4 broad groups: feefor-service, noncapitated models, capitated models or other. A brief description of each model is provided in Table 1. The fee-for-service group includes physicians providing traditional "full-service" primary care and a heterogeneous mixture of "other" fees-for-service, including physicians who practice a formal specialty in addition to family medicine, locums, physician-specialists whose practices focus on such areas as sports medicine or psychotherapy, and physicians covered by the Emergency Department Alternative Framework Agreement who are primarily paid per diems.

The noncapitated models include Family Health Groups, which are paid mainly fee-for-service, plus incentives and bonuses, and a small fee for voluntarily rostering patients; Comprehensive Care Management, which is similar to a Family Health Group in its payment mechanism, except it is not a group model; and physicians in the Rural and Northern Physician Group Agreement, the Group Health Centre, Community Sponsored Agreements, and the Weeneebayko Area Health Authority. Rural models tend to be distinct from the other noncapitated models because their remuneration is more complex and is often not fee-for-service (because their rural location may limit the number of patients in the practice).

The capitated models (where patient rostering is required) include Family Health Organizations, which are paid capitation blended with fee-for-service, together with incentive and bonus components, and Family Health Networks, which are similar to Family Health Organizations but with a smaller capitation component (i.e., a smaller basket of capitated services).

We classify physicians affiliated with the same primary care model all year in model-specific categories. Although physicians can be affiliated with a single model at a time, some physicians changed models during the study period. Physicians who changed between Comprehensive Care Management and Family Health Groups, or between Family Health Networks and Family Health Organizations, were separately identified. All other switchers, and all remaining physicians in a structured payment model, were classified in the "other" category. Physicians not affiliated with any primary care model all year were labelled as traditional fee-for-service. Physicians doing locums and having focused practices were identified as "other" fee-for-service.

Patients are assigned to (perhaps multiple) physicians based on having been seen or rostered. This excludes OHIP beneficiaries who were neither rostered nor saw any physician during the study period.

International medical graduates were defined as physicians who obtained their medical degrees outside of Canada and the United States. ${ }^{7}$ Three rurality categories were defined using the Rurality Index of Ontario as follows: urban (rurality index 0), semiurban (rurality index 1-39), and rural and remote (rurality index $\geq 40$ ). ${ }^{8}$ The number of days worked was calculated based on the number of days on which a physician provided at least 1 service (either fee-for-service and shadow claims).

We identified a patient visit as all claims submitted by the same physician for the same patient on the same day. For the analysis of primary care services (Tables 2-5), we excluded laboratory services (fee codes beginning with an L), unscheduled services (fee code A888A), and emergency department services (fee codes beginning with an $\mathrm{H}$ ). For the analysis of total services (Table 6), we decomposed total services provided into 3 categories: primary care services (all services in the Family Health Organization basket of capitated services); unscheduled visits and emergency or emergency-equivalent services; and all other nonlaboratory services provided by physicians.

Although this study is (statistically) framed from the physician's perspective, we classified patients into 5 mutually exclusive enrolment categories (from the patient's perspective). For each enrolment category, we further classified patients into 6 mutually exclusive patient visit groups based on their formal enrolment status and observed pattern of visits with all physicians. The patient classifications were then tabulated from the physician's perspective to illustrate the nature of the patient population seen by those physicians in each model.

\section{Statistical analyses}

Our descriptive analyses include the number (and percentage), mean, minimum, maximum, median and standard deviation. Because the data contain the entire physician population of Ontario, the unadjusted descriptive analyses should not be interpreted as estimates. Therefore, $95 \%$ confidence intervals are not reported. We performed analyses using Stata 14. 


\section{Ethics approval}

The research was approved by the Hamilton Integrated Research Ethics Board (\#11-086-C).

\section{Results}

Our final sample included 11626 family physicians. Table 2 presents the characteristics of physicians by primary care model. The 3 most popular models (by physician headcount) were traditional fee-for-service (29.1\%), Family Health Groups (23.8\%) and Family Health Organizations (23.7\%). Physicians' mean age was 51.3 years, $38 \%$ were female and $24 \%$ were international medical graduates. Younger physicians were in capitated models (50.6 years old), whereas older physicians were in noncapitated models (52.7 years old).
There were fewer female physicians in fee-for-service (36\%) than in capitated models (40\%). A much lower share (15\%) of physicians in capitated models were international medical graduates relative to noncapitated models (33\%). The average physician billed 206 days per year, with physicians in capitated (236 d) and noncapitated (237 d) models billing more days per year than physicians in fee-for-service $(160 \mathrm{~d})$. The mean group size, for group practices, was by far the highest for Family Health Groups (59.7), followed by Family Health Organizations (16.1) and Family Health Networks (10.4). Most of the physicians in fee-for-service and noncapitated models were in urban areas. No physicians in capitated models were in urban areas; most were in semiurban areas. Given the heterogeneity within the rural models, it is hard to draw any strong conclusions regarding them in this study.

Table 1: Descriptions of primary care models

Model and description

Group size

Payment details

\section{Fee-for-service models}

Traditional

• "Full-service" primary care billing

Solo Fees as per the Ontario Schedule of Benefits for Physician Services

Other

Solo

Fees as per the Ontario Schedule of Benefits for

- Locums Physician Services

- General practitioners whose practices focus on such areas as

sports medicine or psychotherapy

- Emergency Department Alternative Framework Agreement

(physicians are primarily paid per diems)

\section{Noncapitated enrolment models}

Family Health Group

Group Fee-for-service

(min. 3) Enrolment fees and premiums for enrolled patients Incentives and bonuses After-hours premium

Comprehensive Care Management

Solo Fee-for-service

Enrolment fees and premiums for enrolled patients Incentives and bonuses

After-hours premiums

Rural models

- Rural and Northern Physician Group Agreement Varies by model

- Group Health Centre

- Community Sponsored Agreements

-Weeneebayko Area Health Authority

\section{Capitated enrolment models}

Family Health Organizations
Group

(min. 3)
Blended capitation for enrolled patients (capitation plus fee-for-service shadow-billing premium for fee codes inside the capitated basket, plus fee-for-service for codes outside it)

Access bonus for capitated services (at risk if patients see physicians outside the rostering group) Fee-for-service for non-enrolled patients Capped fee-for-service for core services to non-enrolled patients Incentives and bonuses

After-hours premium

Family Health Networks
Similar to Family Health Organizations, but the size of the capitated basket is smaller

\section{Other}

All other physicians not in one of the previously listed models 


\section{OPEN}

Research

Table 2: Physician characteristics by primary care model, 2010/11

\begin{tabular}{|c|c|c|c|c|c|c|c|c|c|c|}
\hline \multirow{2}{*}{$\begin{array}{l}\text { Primary care model } \\
\text { categories }\end{array}$} & \multirow{2}{*}{$\begin{array}{c}\text { No. } \\
\text { (col. \%) }\end{array}$} & \multirow{2}{*}{$\begin{array}{l}\text { Mean } \\
\text { age }\end{array}$} & \multirow{2}{*}{$\begin{array}{l}\text { No. female } \\
(\%)\end{array}$} & \multirow{2}{*}{$\begin{array}{l}\text { No. IMG } \\
(\%)\end{array}$} & \multirow{2}{*}{$\begin{array}{l}\text { Mean no. of } \\
\text { group FPs } \\
\text { interacted with }\end{array}$} & \multirow{2}{*}{$\begin{array}{l}\text { Mean } \\
\text { days } \\
\text { billed }\end{array}$} & \multirow{2}{*}{$\begin{array}{l}\text { No. }(\%) \\
\text { part-time } \\
\text { FPs }\end{array}$} & \multicolumn{3}{|c|}{$\begin{array}{l}\text { No. of FPs by RIO category } \\
(\%)\end{array}$} \\
\hline & & & & & & & & Urban & Semiurban & Rural \\
\hline \multicolumn{11}{|l|}{ Fee-for-service models } \\
\hline Traditional & $\begin{array}{l}3382 \\
(29.1)\end{array}$ & 53.3 & $\begin{array}{c}1059 \\
(31)\end{array}$ & $\begin{array}{l}815 \\
(24)\end{array}$ & 0 & 169 & $\begin{array}{l}782 \\
(23)\end{array}$ & $\begin{array}{l}1939 \\
(57)\end{array}$ & $\begin{array}{l}1247 \\
(37)\end{array}$ & $\begin{array}{l}196 \\
(6)\end{array}$ \\
\hline $\begin{array}{l}\text { Other } \\
\text { (locums and } \\
\text { focused-practice FPs) }\end{array}$ & $\begin{array}{l}705 \\
(6.1)\end{array}$ & 43.1 & $\begin{array}{l}399 \\
(57)\end{array}$ & $\begin{array}{l}109 \\
(15)\end{array}$ & 0 & 113 & $\begin{array}{l}332 \\
(47)\end{array}$ & $\begin{array}{l}327 \\
(46)\end{array}$ & $\begin{array}{l}269 \\
(38)\end{array}$ & $\begin{array}{l}109 \\
(15)\end{array}$ \\
\hline Subtotal & $\begin{array}{l}4087 \\
(35.2)\end{array}$ & 51.5 & $\begin{array}{c}1458 \\
(36)\end{array}$ & $\begin{array}{l}924 \\
(23)\end{array}$ & 0 & 160 & $\begin{array}{l}1114 \\
(27)\end{array}$ & $\begin{array}{c}2266 \\
(55)\end{array}$ & $\begin{array}{l}1516 \\
(37)\end{array}$ & $\begin{array}{c}305 \\
(7)\end{array}$ \\
\hline \multicolumn{11}{|c|}{ Noncapitated enrolment models } \\
\hline Family Health Groups & $\begin{array}{l}2768 \\
(23.8)\end{array}$ & 52.6 & $\begin{array}{l}1066 \\
(39)\end{array}$ & $\begin{array}{l}927 \\
(33)\end{array}$ & 59.7 & 237 & $\begin{array}{l}65 \\
(2)\end{array}$ & $\begin{array}{l}1582 \\
(57)\end{array}$ & $1119(40)$ & $\begin{array}{l}67 \\
(2)\end{array}$ \\
\hline $\begin{array}{l}\text { Comprehensive Care } \\
\text { Management }\end{array}$ & $\begin{array}{l}279 \\
(2.4)\end{array}$ & 56.0 & $\begin{array}{c}83 \\
(30)\end{array}$ & $\begin{array}{l}115 \\
(41)\end{array}$ & 0.0 & 241 & $\begin{array}{c}1 \\
(0)\end{array}$ & $\begin{array}{l}153 \\
(55)\end{array}$ & $\begin{array}{l}96 \\
(34)\end{array}$ & $\begin{array}{c}30 \\
(11)\end{array}$ \\
\hline Rural models & $\begin{array}{r}179 \\
(1.5)\end{array}$ & 49.9 & $\begin{array}{c}59 \\
(33)\end{array}$ & $\begin{array}{c}22 \\
(12)\end{array}$ & 8.8 & 235 & $\begin{array}{c}5 \\
(3)\end{array}$ & $\begin{array}{l}25 \\
(14)\end{array}$ & $\begin{array}{c}22 \\
(12)\end{array}$ & $\begin{array}{l}132 \\
(74)\end{array}$ \\
\hline $\begin{array}{l}\text { Comprehensive Care } \\
\text { Management and } \\
\text { Family Health Groups }\end{array}$ & $\begin{array}{c}99 \\
(0.9)\end{array}$ & 50.1 & $\begin{array}{c}42 \\
(42)\end{array}$ & $\begin{array}{l}38 \\
(38)\end{array}$ & 32.2 & 235 & $\begin{array}{l}3 \\
(3)\end{array}$ & $\begin{array}{l}35 \\
(35)\end{array}$ & $\begin{array}{c}54 \\
(55)\end{array}$ & $\begin{array}{c}10 \\
(10)\end{array}$ \\
\hline Subtotal & $\begin{array}{l}3325 \\
(28.6)\end{array}$ & 52.7 & $\begin{array}{l}1250 \\
(38)\end{array}$ & $\begin{array}{l}1102 \\
(33)\end{array}$ & 51.2 & 237 & $\begin{array}{l}74 \\
(2)\end{array}$ & $\begin{array}{l}1795 \\
(54)\end{array}$ & $\begin{array}{l}1291 \\
(39)\end{array}$ & $\begin{array}{c}239 \\
(7)\end{array}$ \\
\hline \multicolumn{11}{|c|}{ Capitated enrolment models } \\
\hline $\begin{array}{l}\text { Family Health } \\
\text { Organizations }\end{array}$ & $\begin{array}{l}2757 \\
(23.7)\end{array}$ & 50.9 & $\begin{array}{l}1094 \\
(40)\end{array}$ & $\begin{array}{l}414 \\
(15)\end{array}$ & 16.1 & 234 & $\begin{array}{l}48 \\
(2)\end{array}$ & $\begin{array}{c}0 \\
(0)\end{array}$ & $\begin{array}{c}2433 \\
(88)\end{array}$ & $\begin{array}{l}324 \\
(12)\end{array}$ \\
\hline Family Health Networks & $\begin{array}{l}312 \\
(2.7)\end{array}$ & 49.2 & $\begin{array}{l}114 \\
(37)\end{array}$ & $\begin{array}{c}36 \\
(12)\end{array}$ & 10.4 & 256 & $\begin{array}{c}3 \\
(1) \\
\end{array}$ & $\begin{array}{c}0 \\
(0)\end{array}$ & $\begin{array}{l}101 \\
(32)\end{array}$ & $\begin{array}{l}211 \\
(68)\end{array}$ \\
\hline $\begin{array}{l}\text { Family Health Networks } \\
\text { and Family Health } \\
\text { Organizations }\end{array}$ & $\begin{array}{c}124 \\
(1.1)\end{array}$ & 49.3 & $\begin{array}{c}59 \\
(48)\end{array}$ & $\begin{array}{c}14 \\
(11)\end{array}$ & 7.8 & 238 & $\begin{array}{c}0 \\
(0)\end{array}$ & $\begin{array}{c}0 \\
(0)\end{array}$ & $\begin{array}{l}110 \\
(89)\end{array}$ & $\begin{array}{c}14 \\
(11)\end{array}$ \\
\hline Subtotal & $\begin{array}{l}3193 \\
(27.5)\end{array}$ & 50.6 & $\begin{array}{l}1267 \\
(40)\end{array}$ & $\begin{array}{l}464 \\
(15)\end{array}$ & 15.2 & 236 & $\begin{array}{l}51 \\
(2)\end{array}$ & $\begin{array}{c}0 \\
(0)\end{array}$ & $\begin{array}{c}2644 \\
(83)\end{array}$ & $\begin{array}{l}549 \\
(17)\end{array}$ \\
\hline Other & $\begin{array}{l}1021 \\
(8.8)\end{array}$ & 47.7 & $\begin{array}{l}488 \\
(48)\end{array}$ & $\begin{array}{l}305 \\
(30)\end{array}$ & 25.1 & 191 & $\begin{array}{l}140 \\
(14)\end{array}$ & $\begin{array}{l}296 \\
(29)\end{array}$ & $\begin{array}{l}594 \\
(58)\end{array}$ & $\begin{array}{l}131 \\
(13)\end{array}$ \\
\hline Total & $\begin{array}{l}11626 \\
(100 \%)\end{array}$ & 51.3 & $\begin{array}{c}4463 \\
(38)\end{array}$ & $\begin{array}{c}2795 \\
(24)\end{array}$ & 21.0 & 206 & $\begin{array}{c}1379 \\
(12)\end{array}$ & $\begin{array}{c}4357 \\
(37)\end{array}$ & $\begin{array}{c}6045 \\
(52)\end{array}$ & $\begin{array}{c}1224 \\
(11)\end{array}$ \\
\hline
\end{tabular}

Table 3 presents the mean number of patients rostered or seen by a physician. Care must be taken in interpreting the total column because fee-for-service models do not roster, rostering is voluntary for the noncapitated models and rostering is a requirement (with some caveats) for capitated models. Including rostered patients who were not seen in the year, the mean practice size was 1888 , with the minimum less than 10 and the maximum 18000 . Average practice size is slightly larger among capitated models (2046 patients) and much larger among noncapitated models (2528 patients). Most patients were rostered in both noncapitated (53.4\%) and capitated $(71.1 \%)$ models. However, the share of patients rostered but not seen is larger in capitated $(21.6 \%)$ than in noncapitated $(14.8 \%)$ models.

Figure 1 shows the distribution of physicians by practice size in increments of 500 patients. The 4 largest primary care models have a somewhat bell-shaped distribution, with most physicians having a practice size between 1500 and 2500 patients. There is a nontrivial percentage $(4.7 \%)$ of physicians with practice sizes exceeding 5000, with most of these physicians affiliated with Family Health Groups.

Table 4 presents the mean number of visits per patient during the study period based on each patient's rostering status. Overall, patients saw a physician 2.8 times. Across all models, rostered patients saw their physician more (2.9-3.0 visits) than nonrostered patients did (2.6 visits). Physicians in noncapitated models saw their patients more than did those in capitated models. Details regarding the average percentage of patients by enrolment category and visit group, for fee-forservice and the 4 most common primary care models, can be found in Appendix 1, available at www.cmajopen.ca/content/ 4/4/E679/supp1/DC1.

Figure 2 plots the distribution of the mean number of visits per patient by physician. Appreciable variation across physicians 
Table 3: Mean number of patients rostered, seen or both

\begin{tabular}{|c|c|c|c|c|}
\hline Primary care model & $\begin{array}{l}\text { Rostered only } \\
\text { (minimum*, } \\
\text { maximum*, median, } \\
\text { SD, row \%) }\end{array}$ & $\begin{array}{c}\text { Seen only } \\
\text { (minimum }^{\star} \text {, maximum } \\
\text { median, } \text {, }\end{array}$ & $\begin{array}{c}\text { Rostered and seen } \\
\text { (minimum*, maximum *, } \\
\text { median, SD, row \%) }\end{array}$ & $\begin{array}{c}\text { Total } \\
\text { (minimum*, } \\
\text { maximum }{ }^{\star}, \text { median, } \\
\text { SD, row \%) }\end{array}$ \\
\hline \multicolumn{5}{|l|}{ Fee-for-service models } \\
\hline Traditional & $-\dagger$ & $\begin{array}{c}1396 \\
(<10,16500,798,1714.2 \\
100)\end{array}$ & $-\dagger$ & $-\dagger$ \\
\hline $\begin{array}{l}\text { Other (locums and } \\
\text { practice-focused FPs) }\end{array}$ & $-\dagger$ & $\begin{array}{c}1021 \\
(<10,10500,667,1145.8 \\
100)\end{array}$ & $-\dagger$ & $-\dagger$ \\
\hline Subtotal & $-\dagger$ & $\begin{array}{c}1332 \\
(<10,16500,762,1636.4 \\
100)\end{array}$ & $-\dagger$ & $-\dagger$ \\
\hline \multicolumn{5}{|l|}{$\begin{array}{l}\text { Noncapitated } \\
\text { enrolment models }\end{array}$} \\
\hline Family Health Groups & $\begin{array}{c}372 \\
(<10,4500,305 \\
328.7,14.1)\end{array}$ & $\begin{array}{c}1277 \\
(<10,17500,616.5 \\
1652.7,48.3)\end{array}$ & $\begin{array}{c}994 \\
(<10,3500,910,640.5,37.6)\end{array}$ & $\begin{array}{c}2643 \\
(<10,18000,2210, \\
1743.8,100)\end{array}$ \\
\hline $\begin{array}{l}\text { Comprehensive Care } \\
\text { Management }\end{array}$ & $\begin{array}{c}364 \\
(<10,1500,298 \\
295.1,17.0)\end{array}$ & $\begin{array}{c}704 \\
(<10,6500,242,1133.0 \\
32.9)\end{array}$ & $\begin{array}{c}1073 \\
(<10,3500,971,614.4,50.1)\end{array}$ & $\begin{array}{c}2142 \\
(400,7500,1730, \\
1320.7,100)\end{array}$ \\
\hline Rural models & $\begin{array}{c}376 \\
(<10,1500,318 \\
303.5,25.6)\end{array}$ & $\begin{array}{c}450 \\
(<10,2500,341,406.7, \\
30.6)\end{array}$ & $\begin{array}{c}644 \\
(<10,2000,616,380.7,43.8)\end{array}$ & $\begin{array}{c}1470 \\
(300,3500,1402 \\
699.6,100)\end{array}$ \\
\hline $\begin{array}{l}\text { Comprehensive Care } \\
\text { Management and Family } \\
\text { Health Groups }\end{array}$ & $\begin{array}{c}436 \\
(<10,1500,358,355, \\
18.6)\end{array}$ & $\begin{array}{c}1117 \\
(<10,4500,694,1117.1, \\
47.7)\end{array}$ & $\begin{array}{c}788 \\
(<10,3000,611,564,33.7)\end{array}$ & $\begin{array}{c}2342 \\
(100,5500,2241 \\
1282.1,100)\end{array}$ \\
\hline Subtotal & $\begin{array}{c}373 \\
(<10,4500,306 \\
325.5,14.8)\end{array}$ & $\begin{array}{c}1179 \\
(<10,17500,549,1575.6 \\
46.6)\end{array}$ & $\begin{array}{c}976 \\
(<10,3500,890,631.1,38.6)\end{array}$ & $\begin{array}{c}2528 \\
(<10,1800,2114, \\
1684.2,100)\end{array}$ \\
\hline \multicolumn{5}{|l|}{$\begin{array}{l}\text { Capitated enrolment } \\
\text { models }\end{array}$} \\
\hline $\begin{array}{l}\text { Family Health } \\
\text { Organizations }\end{array}$ & $\begin{array}{c}441 \\
(<10,2500,406 \\
252.2,21.2)\end{array}$ & $\begin{array}{c}595 \\
(<10,1000,458,597.2, \\
28.5)\end{array}$ & $\begin{array}{c}1048 \\
(<10,2500,1025,467.2, \\
50.3)\end{array}$ & $\begin{array}{c}2085 \\
(<10,12000,1999, \\
908.8,100)\end{array}$ \\
\hline Family Health Networks & $\begin{array}{c}364 \\
(<10,1000,334 \\
199.9,20.4)\end{array}$ & $\begin{array}{c}627 \\
(<50,4000,494.5,536.9 \\
35.1)\end{array}$ & $\begin{array}{c}794 \\
(<10,2000,736.5,330.9 \\
44.5)\end{array}$ & $\begin{array}{c}1785 \\
(<150,5000,1675, \\
719.0,100)\end{array}$ \\
\hline $\begin{array}{l}\text { Family Health Networks } \\
\text { and Family Health } \\
\text { Organizations }\end{array}$ & $\begin{array}{c}673(<20,2000,604 \\
346.2,36.3)\end{array}$ & $\begin{array}{c}402 \\
(<50,3500,353,381.1, \\
21.6)\end{array}$ & $\begin{array}{c}785 \\
(<10,2000,734,338.6,42.2)\end{array}$ & $\begin{array}{c}1860 \\
(<1000,6500,1707, \\
731.8,100)\end{array}$ \\
\hline Subtotal & $\begin{array}{c}443 \\
(<10,2500,403 \\
257.1,21.6)\end{array}$ & $\begin{array}{c}591 \\
(<10,10000,455,585.8 \\
28.9)\end{array}$ & $\begin{array}{c}1013 \\
(<10,2500,969,459.7,49.5)\end{array}$ & $\begin{array}{c}2046 \\
(<10,12000,1941, \\
890.8,100)\end{array}$ \\
\hline Other & $\begin{array}{c}297 \\
(<10,3500,165 \\
378.7,19.4)\end{array}$ & $\begin{array}{c}723 \\
(<10,7500,365,951.4, \\
47.2)\end{array}$ & $\begin{array}{c}512 \\
(<10,2500,378,493.7,33.4)\end{array}$ & $\begin{array}{c}1532 \\
(<10,10000,1275, \\
1121.4,100)\end{array}$ \\
\hline Total & $\begin{array}{c}254 \\
(<10,4500,178 \\
312.6,13.5)\end{array}$ & $\begin{array}{c}1031 \\
(<10,17500,548.5 \\
1387.2,54.6)\end{array}$ & $\begin{array}{c}602 \\
(<10,3500,504,638.6,31.9)\end{array}$ & $\begin{array}{c}1888 \\
(<10,18000 \\
1610.5,1524.7,100)\end{array}$ \\
\hline
\end{tabular}




\section{OPEN}

Research

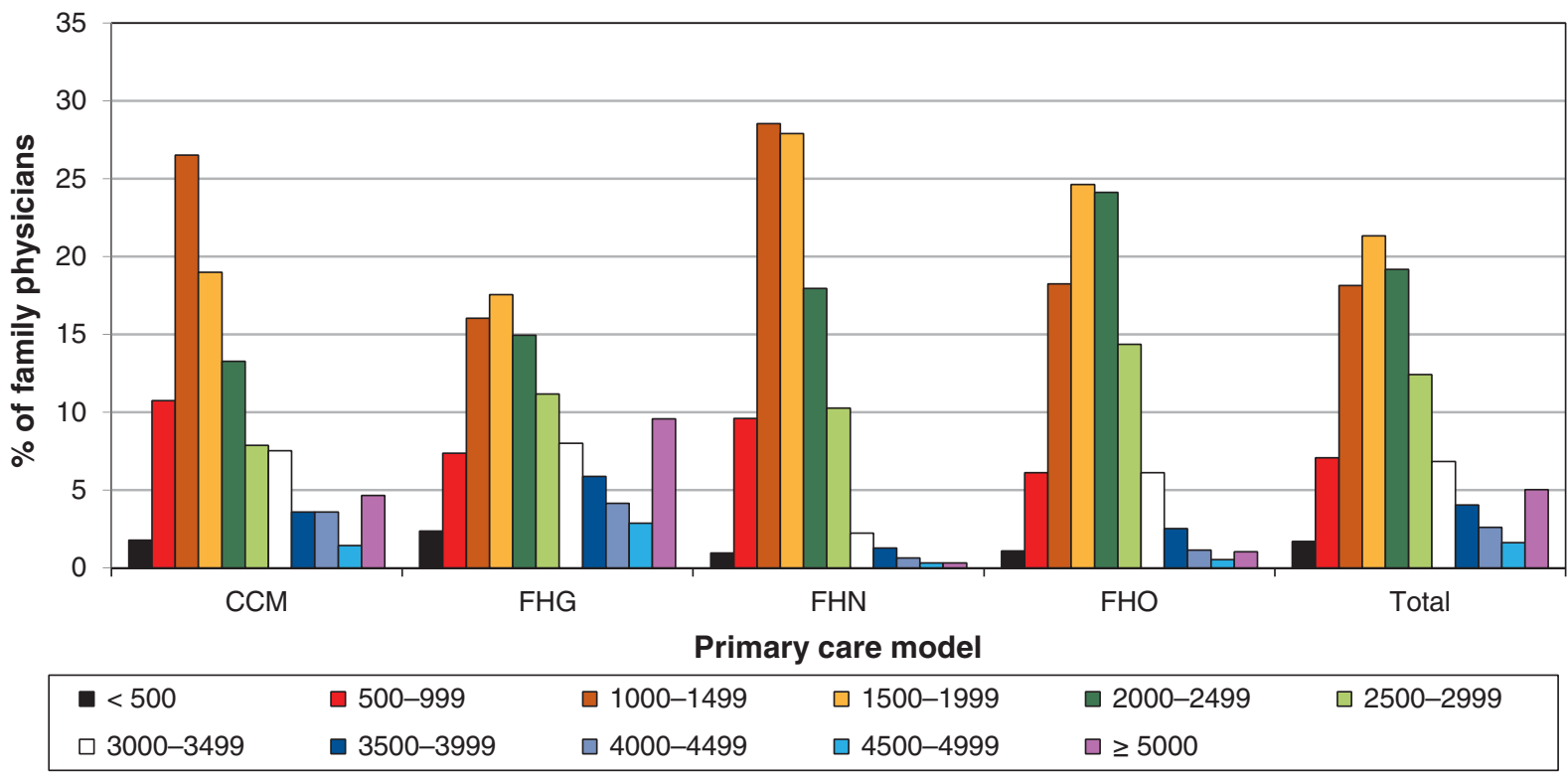

Figure 1: Percentage of physicians by number of patients per practice, selected primary care models. CCM = Comprehensive Care Management, FHG = Family Health Group, FHN = Family Health Network, FHO = Family Health Organization.

Table 4: Visits per patient, by patient category

\begin{tabular}{|c|c|c|c|c|}
\hline \multirow[b]{2}{*}{ Primary care model } & \multicolumn{4}{|c|}{ No. of visits, mean (SD) } \\
\hline & Rostered all year & Rostered < year & Not rostered & Total \\
\hline \multicolumn{5}{|l|}{ Fee-for-service models } \\
\hline Traditional & NA & NA & $3.5(4.3)$ & NA \\
\hline Other (locums and focused-practice FPs) & NA & NA & $1.8(1.8)$ & NA \\
\hline Subtotal & NA & NA & $3.2(4.0)$ & NA \\
\hline \multicolumn{5}{|l|}{ Noncapitated enrolment models } \\
\hline Family Health Groups & $3.4(1.8)$ & $3.1(1.8)$ & $2.5(1.8)$ & $3.0(1.5)$ \\
\hline Comprehensive Care Management & $3.9(1.5)$ & $3.2(1.6)$ & $3.0(1.7)$ & $3.6(1.4)$ \\
\hline Rural models & $2.5(1.0)$ & $2.4(1.2)$ & $2.4(2.5)$ & $2.4(1.0)$ \\
\hline $\begin{array}{l}\text { Comprehensive Care Management and Family Health } \\
\text { Groups }\end{array}$ & $3.4(1.6)$ & $3.2(1.8)$ & $2.1(1.1)$ & $2.7(1.3)$ \\
\hline Subtotal & $3.4(1.8)$ & $3.0(1.8)$ & $2.5(1.8)$ & $3.0(1.5)$ \\
\hline \multicolumn{5}{|l|}{ Capitated enrolment models } \\
\hline Family Health Organizations & $2.6(1.2)$ & $2.8(1.4)$ & $2.1(1.1)$ & $2.4(1.0)$ \\
\hline Family Health Networks & $2.8(1.3)$ & $3.3(1.6)$ & $2.0(1.0)$ & $2.5(1.0)$ \\
\hline $\begin{array}{l}\text { Family Health Networks and Family Health } \\
\text { Organizations }\end{array}$ & $2.4(0.7)$ & $2.7(1.1)$ & $2.1(1.3)$ & $2.2(0.7)$ \\
\hline Subtotal & $2.6(1.2)$ & $2.8(1.4)$ & $2.1(1.1)$ & $2.4(1.0)$ \\
\hline Other & $2.8(1.0)$ & $2.6(1.6)$ & $1.9(1.3)$ & $2.2(1.1)$ \\
\hline Total & $3.0(1.5)$ & $2.9(1.6)$ & $2.6(2.7)$ & $2.8(2.6)$ \\
\hline
\end{tabular}


in the mean number of times they see each patient can be seen. Between $71 \%$ and $87 \%$ of physicians in primary care models saw their patients between 2 and 5.9 times each during the study period, whereas one-third of traditional fee-for-service physicians are in this group. Traditional fee-for-service physicians were more polarized, with a very high percentage in the $1-1.9$ visit category and a substantial percentage at the other end of the spectrum. Of all physicians seeing their patients 6 or more times, $82 \%$ were in the traditional fee-for-service payment model.

One key goal of both rostering and capitation is to foster a relationship between the patient and their rostering physician (and the rostering group, who share medical records). Table 5 compares the mean percentage of patients in a physician's practice who saw only 1 physician during the year. For the system overall, there was only a modest increase, from $25.8 \%$ to $28.7 \%$, in "unique visit patterns" between 2000/01 and $2010 / 11$. However, there is diversity in $2010 / 11$ between traditional fee-for-service $(14.2 \%)$ and the 4 most common models (33\% to $48 \%$ ).

Figure 3 shows the percentage of patients in a practice by the 5 rostering categories, as seen in Appendix 1, for Comprehensive Care Management, Family Health Groups, Family

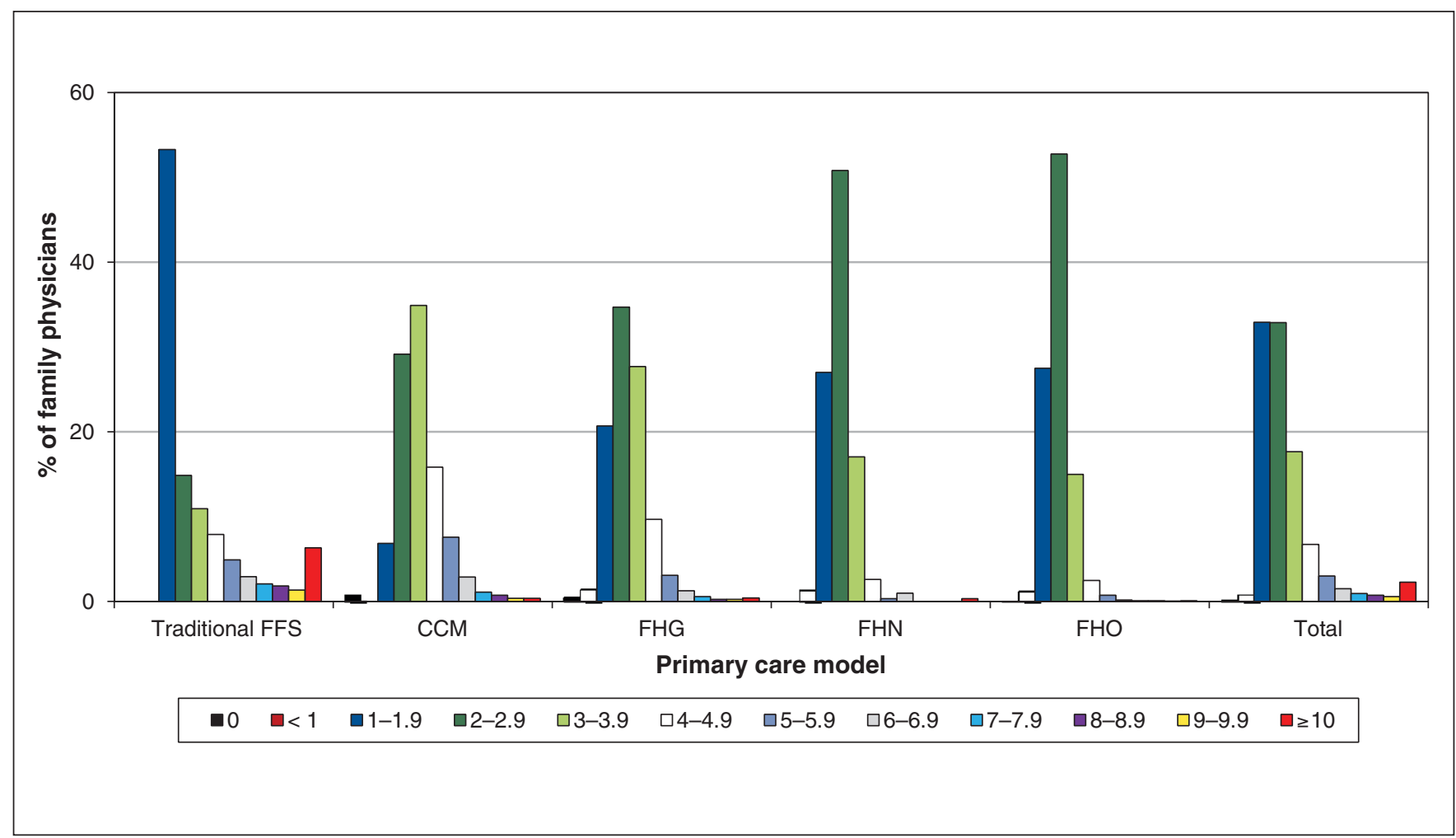

Figure 2: Percentage of physicians by mean number of visits* per patient, selected primary care models. *Visits related to primary care or other services only (i.e., unscheduled and emergency services are excluded). CCM $=$ Comprehensive Care Management, FFS = fee-for-service, FHG $=$ Family Health Group, FHN = Family Health Network, FHO = Family Health Organization.

Table 5: Average percentage of patients in a physician's practice who saw one physician in the year, selected primary care models

\begin{tabular}{|c|c|c|c|c|c|c|}
\hline Fiscal year & All FPs, \% & FFS, \% & CCM, \% & FHG, \% & FHN, \% & $\mathrm{FHO}^{*}, \%$ \\
\hline $2000 / 01$ & $\begin{array}{c}25.8 \\
\text { (4 } 100387 \text { patients overall) }\end{array}$ & 25.4 & & $\begin{array}{c}\text { Not applicable } \\
\text { (model did not exist) }\end{array}$ & & 56.8 \\
\hline $2010 / 11$ & $\begin{array}{c}28.7 \\
\text { (5 } 264305 \text { patients overall) }\end{array}$ & 14.2 & 47.7 & 32.9 & 39.5 & 41.8 \\
\hline
\end{tabular}




\section{OPEN}

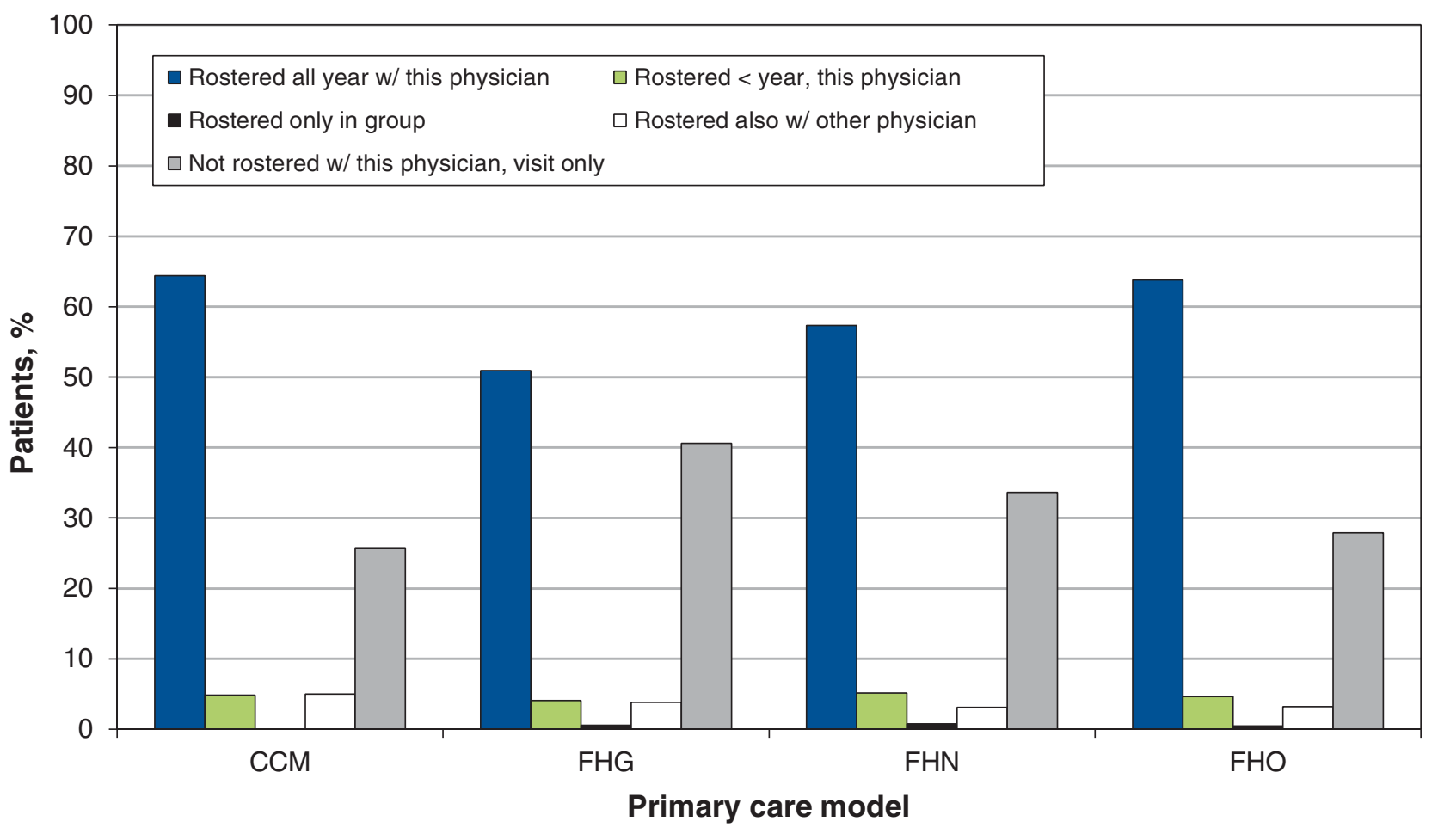

Figure 3: Percentage of patients by patient enrolment category, selected primary care models. CCM = Comprehensive Care Management, $\mathrm{FHG}=$ Family Health Group, FHN = Family Health Network, FHO = Family Health Organization.

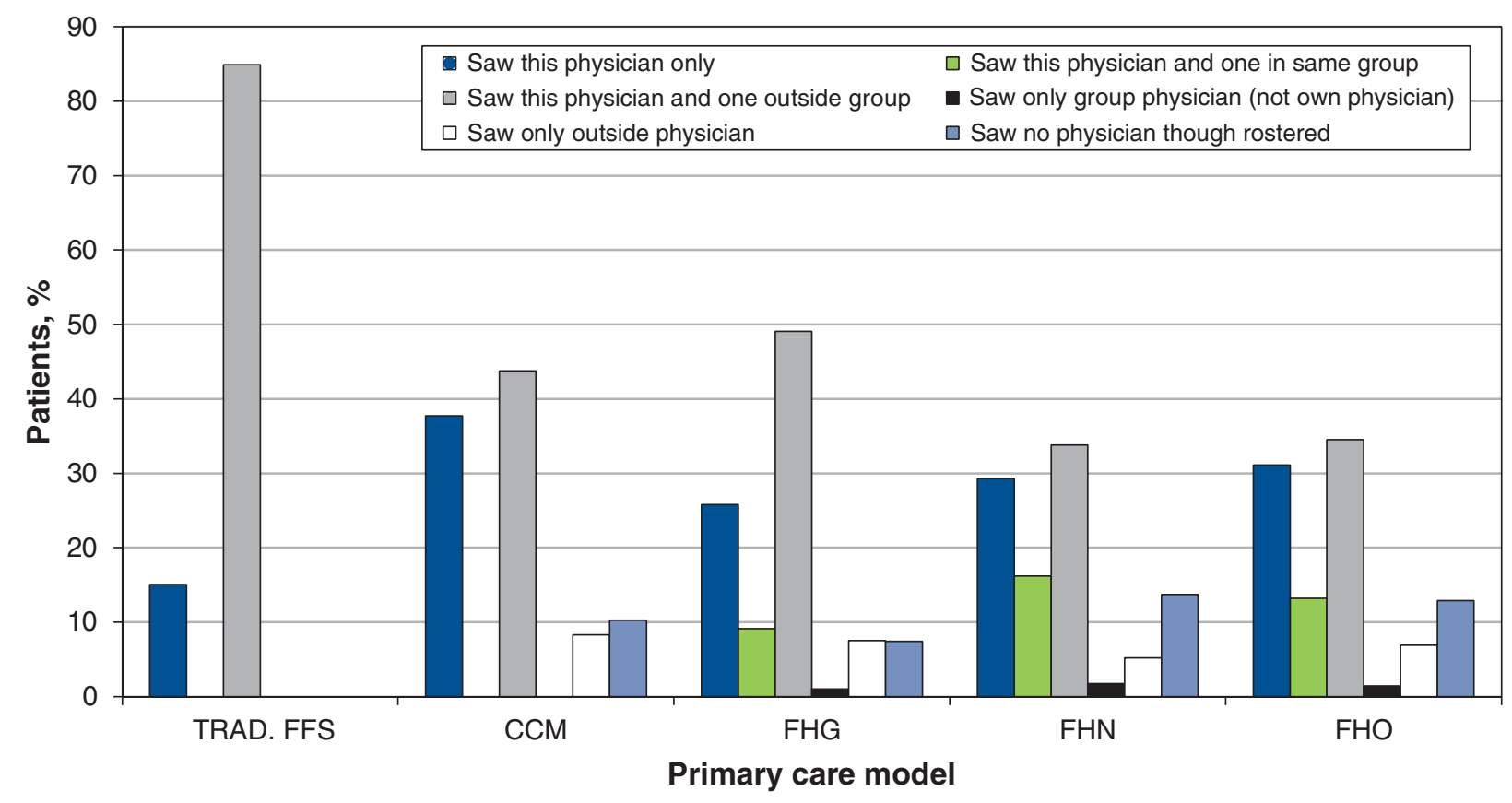

Figure 4: Percentage of patients by visit group, selected primary care models. $\mathrm{CCM}=$ Comprehensive Care Management, $\mathrm{FHG}=\mathrm{Family}$ Health Group, Trad. FFS = traditional fee-for-service, FHN = Family Health Network, FHO = Family Health Organization 


\begin{tabular}{|c|c|c|c|}
\hline Primary care model & $\begin{array}{c}\text { Primary care } \\
\text { services, } \\
\%\end{array}$ & $\begin{array}{c}\text { Unscheduled or emergency } \\
\text { services, } \\
\%\end{array}$ & $\begin{array}{l}\text { Other services, } \\
\%\end{array}$ \\
\hline \multicolumn{4}{|l|}{ Fee-for-service models } \\
\hline Traditional & 53.9 & 14.2 & 31.9 \\
\hline Other & 59.5 & 16.9 & 23.5 \\
\hline Subtotal & 54.9 & 14.7 & 30.5 \\
\hline \multicolumn{4}{|l|}{ Non-capitated enrolment models } \\
\hline Family Health Groups & 75.1 & 3.0 & 21.9 \\
\hline Comprehensive Care Management & 76.4 & 2.0 & 21.5 \\
\hline Rural models & 63.3 & 5.0 & 31.7 \\
\hline $\begin{array}{l}\text { Comprehensive Care Management and Family } \\
\text { Health Groups }\end{array}$ & 77.0 & 2.6 & 20.4 \\
\hline Subtotal & 74.6 & 3.1 & 22.3 \\
\hline \multicolumn{4}{|l|}{ Capitated enrolment models } \\
\hline Family Health Organizations & 67.8 & 3.4 & 28.8 \\
\hline Family Health Networks & 56.7 & 9.6 & 33.7 \\
\hline $\begin{array}{l}\text { Family Health Networks and Family Health } \\
\text { Organizations }\end{array}$ & 64.5 & 4.8 & 30.7 \\
\hline Subtotal & 66.6 & 4.1 & 29.3 \\
\hline Other & 68.8 & 5.1 & 26.1 \\
\hline Total & 64.9 & 7.6 & 27.5 \\
\hline
\end{tabular}

Health Networks and Family Health Organizations. The largest category of patients in the practice is patients rostered all year with the physician.

Figure 4 plots the percentage of patients in a practice by the 6 major visit groups described in Appendix 1. In the newer primary care models, there was a greater share of patients who visit only 1 physician compared with traditional fee-for-service. However, primary care models display a low percentage of patients who visit only their rostering physician or a physician in the same group. Across all primary care models, a large share of patients visit their rostering physician and a physician outside the rostering group.

Table 6 breaks down total patient visits into 3 categories. Noncapitated primary care models (Comprehensive Care Management and Family Health Groups) provided the highest percentage of primary care services, whereas fee-for-service provided the lowest. Interestingly, models with the lowest percentage of primary care visits also had smaller practice sizes.

\section{Interpretation}

Diversity exists across primary care models. Younger physicians and female physicians are slightly more likely, and international medical graduates are much less likely, to practice in a capitated model (Family Health Organization and Family Health Network). In addition, we found substantial geographic differences, with no capitated models found in urban areas. The noncapi- tated models (specifically Family Health Groups) have large practice sizes compared with other models. Among patients with multiple visits, primary care models have low percentages of patients visiting the same physician (or a physician within the same group) repeatedly, suggesting little within-group coverage, which is remarkable considering patient visits did not include unscheduled and emergency services.

The results suggest primary care reform in Ontario has successfully shifted physicians away from fee-for-service toward the newer primary care models. Most physicians who provide more traditional family medicine and who have a unique relationship with their patients (with respect to visit frequency and visit group) have transitioned to the newer models. Physicians who are relatively less active, have multiple specialties, are locums or have more unusual relationships with patients in regards to visit frequency have remained in the fee-for-service model. The fee-for-service model is somewhat polarized, with many physicians at one end of the spectrum - working fewer days per year, having small practices and seeing patients only once. Conversely, others see their patients more than 6 times a year. The Family Health Group and Comprehensive Care Management noncapitated models attract physicians with the largest practices.

Physicians in most primary care models have rostered 50\%$70 \%$ of patients. However, most models contain a high percentage of patients who see physicians but are rostered neither with 
them nor with their group, which is more surprising for the capitated models than the non-capitated models (where rostering is voluntary). From the perspective of group integration and continuity of primary care, group-based models do not appear to be making a large impact. The Family Health Group model has the lowest percentage of patients rostered all year who saw their physician only, and the highest percentage of patients not rostered with a physician who saw both a group physician and a nongroup physician. Despite continuity being associated with high-quality care, ${ }^{9-13}$ recent research has emphasized how continuity is threatened by limited access to specialized or readily available care. ${ }^{9}, 14,15$

\section{Limitations}

The main limitation of our study is the age of the data (2010/11). The results for more recent years may be different, given the movement of physicians across primary care models. In addition, we only focused on physician and patient characteristics across primary care models, because the administrative data do not contain information on patient health. Although we have focused on the variation in physician and patient characteristics across different models, we cannot indicate how these variations may affect other aspects of the health care system, such as referrals to specialists, diagnostic testing or prescribing patterns.

\section{Conclusion}

Ontario's new primary care models appear to have been successful both in moving many physicians away from traditional fee-for-service payment models, and in enrolling the majority of Ontario patients with a physician. However, our results also suggest group-based models may not be having a large impact on team-based coverage with respect to the provision of primary care services. There also appears to be heterogeneity across practices with respect to the number of patients seen, geographic uptake (as seen by Family Health Organizations being located outside urban locations) and some physician demographics. Further research is needed on the effects of changes in primary care on patient health.

\section{References}

1. Sweetman A, Buckley G. Ontario's experiment with primary care reform. University of Calgary School of Public Policy Research Paper 7(11); 2014. Available: www.policyschool.ca/wp-content/uploads/2016/03/ontario-health-care-reform.pdf (accessed 2015 Dec. 16)

2. Glazier RH, Zagorski BM, Rayner J. Comparison of primary care models in Ontario: by demographics, case mix and emergency department use, 2008/09 to 2009/10 - ICES Investigative Report. Toronto: Institute for Clinical Evaluative
Sciences; 2012. Available: www.ices.on.ca/Publications/Atlases-and-Reports/2012/ Comparison-of-Primary-Care-Models (accessed 2015 Dec. 16).

3. Family practice models. Toronto: HealthForceOntario; 2013. Available: www. healthforceontario.ca/en/Home/Physicians/Training_\%7C_Practising_in_Ontario/ Physician_Roles/Family_Practice_Models (accessed 2014 Dec. 19).

4. Sweetman A, Buckley G. Better ways to pay for health care. Paris: OECD Publishing; 2016. Available: www.oecd.org/health/better-ways-to-pay-for-health-care9789264258211-en.htm (accessed 2016 July 18).

5. Rudoler D, Deber R, Barnsley J, et al. Paying for primary care: the factors associated with physician self-selection into payment models. Health Econ 2015;24: 1229-42.

6. Rudoler D, Laporte A, Barnsley J, et al. Paying for primary care: a cross-sectional analysis of cost and morbidity distributions across primary care payment models in Ontario Canada. Soc Sci Med 2015;124:18-28.

7. Barer ML, Stoddart GL. Toward integrated medical resource policies for Canada: 4. Graduates of foreign medical schools. CMA7 1992;146:1549-54.

8. Kralj B. Measuring "rurality" for purposes of health care planning: an empirical measure for Ontario. Ont Med Rev 2000;67:33-52.

9. Haggerty JL, Reid RJ, Freeman GK, et al. Continuity of care: a multidisciplinary review. BM7 2003;327:1219-21.

10. Hjortdahl P, Laerum E. Continuity of care in general practice: effect on patient satisfaction. BM7 1992;304:1287-90.

11. Ionescu-Ittu R, McCusker J, Ciampi A, et al. Continuity of primary care and emergency department utilization among elderly people. CMA7 2007; 177:1362-8.

12. Menec VH, Sirski M, Attawar D. Does continuity of care matter in a universally insured population? Health Serv Res 2005;40:389-400.

13. Primary care: putting people first. In: Evans T, Van Lerberghe W, editors. The World Health Report 2008: primary health care - now more than ever. Geneva: World Health Organization; 2008. Available: www.who.int/whr/2008/08_ chap3_en.pdf?ua=1 (accessed 2014 Dec. 17).

14. Guthrie B, Saultz JW, Freeman GK, et al. Continuity of care matters. BMF 2008;337:a867.

15. Rosser $W$, Schultz K. Promoting continuity of care should be integral to any health care system. CMA7 2007;177:1385-6.

16. Hutchison B, Glazier RH. Ontario's primary care reforms have transformed the local care landscape, but a plan is needed for ongoing improvement. Health Aff (Millwood) 2013;32:695-703.

Affiliations: Lazaridis School of Business \& Economics (McLeod), Wilfrid Laurier University, Waterloo, Ont.; Centre for Health Economics and Policy Analysis (Buckley) and Department of Economics (Sweetman), McMaster University, Hamilton, Ont.

Contributors: Logan McLeod was responsible for some of the data analysis, contributed to the interpretation of the results and drafted parts of the manuscript. Gioia Buckley was responsible for acquiring the data, conducted most of the data analysis and drafted some sections of the manuscript. Arthur Sweetman was responsible for the concept and design of the study, was responsible for acquiring the data, contributed to the interpretation of the results, and provided input on various drafts of the manuscript. All of the authors approved the final version to be published and agreed to act as guarantors of the results.

Funding: This manuscript was funded by the Government of Ontario and by Ontario SPOR SUPPORT Unit (OSSU), which is supported by the Canadian Institutes of Health Research and the Province of Ontario. The views expressed in the manuscript are the views of the authors and should not be taken to represent the views of OSSU nor of the Government of Ontario.

Supplemental information: For reviewer comments and the original submission of this manuscript, please see www.cmajopen.ca/content/4/4/ E679/suppl/DC1 